\title{
A Study of High Frequency Ultrasound Scattering from Non- Nucleated Biological Specimens
}

\section{Omar Falou}

Ryerson University

\section{Ralph E. Baddour}

University of Toronto

\section{George Nathanael}

University of Toronto

\section{Gregory J. Czarnota}

Toronto Sunnybrook Regional Cancer Centre

\section{J. Carl Kumaradas}

Ryerson University

\section{digital.library.ryerson.ca/object/339}

\section{Please Cite:}

Falou, O., Baddour, R. E., Nathanael, G., Czarnota, G. J., Kumaradas, J. C., \& Kolios, M. C. (2008). A study of high frequency ultrasound scattering from nonnucleated biological specimens. The Journal of the Acoustical Society of America, 124(5), EL278-EL283.

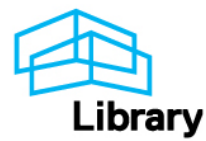




\title{
A study of high frequency ultrasound scattering from non-nucleated biological specimens
}

\author{
Omar Falou \\ Department of Electrical and Computer Engineering, Ryerson University, 350 Victoria Street, Toronto, \\ Ontario M5B 2K3, Canada \\ ofalou@ryerson.ca \\ Ralph E. Baddour and George Nathanael \\ Department of Medical Biophysics, University of Toronto, 610 University Avenue, Toronto, \\ Ontario M5G 2M9, Canada \\ rbaddour@uhnres.utoronto.ca, \\ gnathana@uwo.ca \\ Gregory J. Czarnota \\ Department of Radiation Oncology, Toronto Sunnybrook Regional Cancer Centre, Toronto, \\ Ontario M4N 3M5, Canada \\ gregory.czarnota@sunnybrook.ca \\ J. Carl Kumaradas and Michael C. Kolios \\ Department of Physics, Ryerson University, 350 Victoria Street, Toronto, Ontario M5B 2K3, Canada \\ ckumarad@ryerson.ca, \\ mkolios@ryerson.ca
}

\begin{abstract}
The high frequency backscatter from cells with a nucleus to cell volume ratio of 0.50 cannot be adequately modeled as a homogeneous sphere. It was hypothesized that the cytoplasm of such cells is of fluid nature. This work attempts to model the ultrasound backscatter (10-62 MHz) from some non-nucleated biological specimens. This was done by measuring the backscatter response from individual sea urchin oocytes and comparing it to theoretical predictions in both the time and frequency domains. A good agreement was found between the experimental and theoretical results suggesting that the non-nucleated oocytes are of fluid nature.

(C) 2008 Acoustical Society of America

PACS numbers: 43.80.Cs, 43.80.Gx [Charles Church]

Date Received: June 17, 2008 Date Accepted: July 30, 2008
\end{abstract}

\section{Introduction}

When compared to clinical ultrasound imaging $(1-10 \mathrm{MHz})$, high frequency ultrasound imaging $(20-60 \mathrm{MHz})$ is more sensitive to cell structure and cell spatial distribution changes. ${ }^{1,2}$ Recent publications have demonstrated that high frequency ultrasound has the potential of being used for tumor classification. ${ }^{3}$ Others have shown that high frequency ultrasound can be used to detect structural and physical changes in cell ensembles during apoptosis. ${ }^{4}$ Ultrasonic backscatter from cell ensembles treated with the chemotherapeutic drug cisplatin, which induces apoptosis (apoptosis is described by Kerr et al. ${ }^{5}$ ), increased the ultrasound backscatter signal amplitude by $9-13 \mathrm{~dB}$ and induced changes in the frequency dependence of backscatter. ${ }^{4,6}$ Theoretical models of ultrasound scattering at the cellular level are needed in order to develop methods for using ultrasound backscatter measurements to classify tumors or determine their response to treatment. The development of these models requires an understanding of the mechanical properties of components of a cell such as the nucleus and the cytoplasm.

Baddour et al. ${ }^{7}$ performed successful measurements of high frequency $(10-65 \mathrm{MHz})$ backscatter from single cells. A recent study by the same group showed that for prostate carci- 


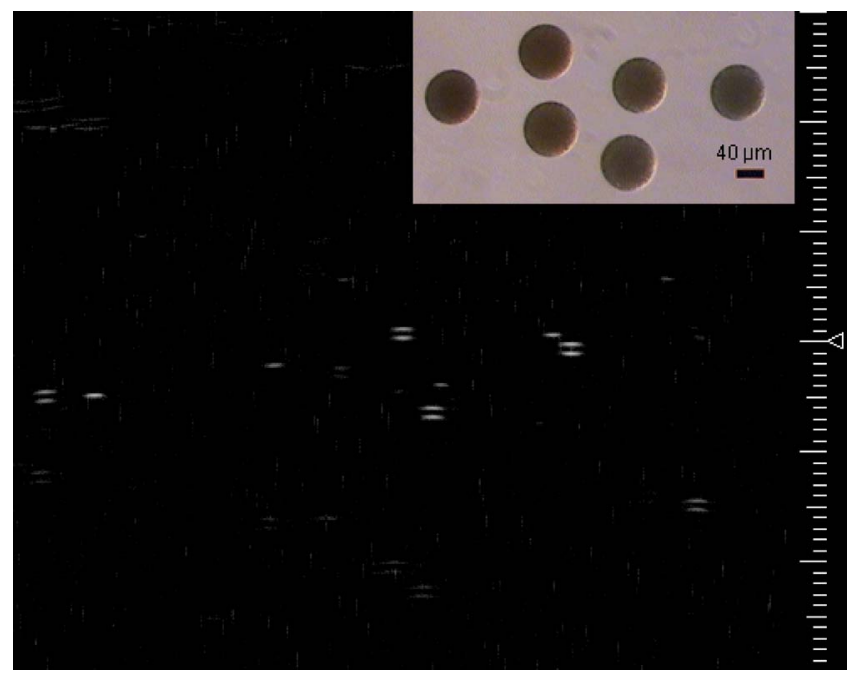

Fig. 1. (Color online) B scan of a sparse suspension of sea urchin oocytes in seawater at $40 \mathrm{MHz}$ (smallest scale $=100 \mu \mathrm{m}$ ). The triangle on the right hand side of the image indicates the location of the transducer focus. Inset is an optical microscopy of sea urchin oocytes.

noma (PC-3) cells whose nucleus to cell volume ratio is 0.33 , the backscatter response could be modeled as a fluid sphere. ${ }^{8}$ However, the same study found that for human acute myeloid leukemia (OCI-AML-5) cells whose nucleus to cell volume ratio equals to 0.50 , the backscatter response was not modeled as well by a fluid sphere. Baddour et al. hypothesized that the cytoplasm is of fluid nature whereas the nucleus possesses elastic properties, giving rise to this discrepancy. ${ }^{8}$ This work attempts to model the ultrasound backscatter (10-62 MHz) from some non-nucleated biological specimens through the measurement and comparison of the backscatter response from sea urchin oocytes to theoretical predictions from a fluid sphere.

\section{Methods}

\subsection{Calculation of backscatter transfer function}

A sparse suspension of oocytes from Strongylocentrotus purpuratus (purple sea urchin) were prepared in artificial seawater $\left(\rho=1025 \mathrm{~kg} / \mathrm{m}^{3}, c=1527 \mathrm{~m} / \mathrm{s}\right){ }^{9}$ The oocytes were obtained by shedding live female urchins into artificial seawater with $0.5 \mathrm{M} \mathrm{KCL}$. They are mainly composed of chromatin and yolk proteins. These oocytes were selected because their spherical shape and narrow size distribution, as shown in the inset of Fig. 1 (mean oocyte diameter of approximately $75 \mu \mathrm{m}$, standard deviation $=2.2 \mu \mathrm{m}$ ).

Data acquisition was performed using a VisualSonics VS40B ultrasound imaging device (VisualSonics Inc., Canada). Three transducers, with different resonant frequencies, f-numbers, and focal lengths, were employed. Table 1 summarizes the properties of the three transducers. Data from the $-6 \mathrm{~dB}$ bandwidth of each spectrum of the incident pulse (as measured by the reflection off a quartz plate) were used in the analysis, giving an overall analysis range of 10-62 MHz. Ten independent acquisitions of 75, 100, and 125 linearly separated (150 $\mu \mathrm{m}$ spacing) raw rf echo signals were performed using the 20,40 , and $80 \mathrm{MHz}$ transducers, respectively. Since oocytes centered in the focal region of a given transducer lead to a higher amplitude echo opposed to oocytes that are located off the transducer axis, any rf scan lines whose maximum was less than $90 \%$ of the overall maximum value from all $\mathrm{rf}$ lines were discarded, assuming they did not contain an oocyte completely in the focal region of that scan. 
Table 1. Properties of the transducers used in the experiments

\begin{tabular}{lcccc}
\hline \hline Transducer & f-number & $\begin{array}{c}\text { Focal length } \\
(\mathrm{mm})\end{array}$ & $\begin{array}{c}\text { Excitation } \\
\text { frequency } \\
(\mathrm{MHz})\end{array}$ & $\begin{array}{c}-6 \mathrm{~dB} \\
\text { bandwidth } \\
(\mathrm{MHz})\end{array}$ \\
\hline $20 \mathrm{MHz}$ polyvinylidene fluoride & 2.35 & 20 & 19 & $10-28$ \\
$40 \mathrm{MHz}$ polyvinylidene fluoride & 3.00 & 9 & 40 & $21-51$ \\
$80 \mathrm{MHz}$ lithium niobate & 3.00 & 6 & $55^{\text {a }}$ & $40-62$ \\
\hline \hline
\end{tabular}

${ }^{\text {a }}$ The $80 \mathrm{MHz}$ transducer was pulsed at $55 \mathrm{MHz}$ in order to expand the frequency band for this study to $62 \mathrm{MHz}$. The performance of the transducer was satisfactory.

A Hamming window of $2 \mu$ s width, centered at the maximum value of each rf line, was applied to all remaining lines in order to partially remove abnormal scattering patterns in some lines due to the presence of more than one oocyte in the focal region (resolution volume) of the scan. Furthermore, visual inspection was performed to eliminate any lines which exhibited these patterns and were not removed by the previous step. For each transducer, the remaining rf lines (of which there were between 5 and 23) were translated to align the midpoint of the two largest positive peaks and then averaged to obtain a single rf line corresponding to the backscatter from an individual "average" oocyte. From this, a backscatter transfer function $\operatorname{BSTF}_{\text {expr }}(\omega)$ was calculated:

$$
\operatorname{BSTF}_{\text {expr }}(\omega)=\frac{R_{\text {expr }}(\omega)}{R_{\text {ref }}(\omega)}
$$

where $R_{\text {expr }}(\omega)$ is the Fourier transform of the average backscatter signal (within the transducer's depth of field). $R_{\text {ref }}(\omega)$ is the Fourier transform of the average reference signal. Reference signals were obtained using the specular reflection from a flat polished $\mathrm{SiO}_{2}$ crystal (Edmund Industrial Optics Inc., part 43424; $\rho=2200 \mathrm{~kg} / \mathrm{m}^{3}, c=5720 \mathrm{~m} / \mathrm{s}$ ) placed at the transducer focus in seawater. The average reference signal was obtained by superimposing and averaging 32 independent acquisitions of rf echo signals for each transducer. The values of the $|\mathrm{BSTF}|^{2}$ are presented in the form of spectral plots expressed in decibels relative to the backscatter intensity from the reference $\left(\mathrm{dB}_{r}\right)$. These were compared with the theoretical backscatter frequency responses calculated for a fluid sphere using the Anderson model, ${ }^{10}$ with a range of densities and sound speeds for the scattering sphere. A least squares analysis was used to determine the density and speed of sound for the fluid sphere that best agreed with the experimental response in the spectral domain. This was performed by minimizing the sum of the squares of the difference between theoretical and experimental values. Densities from 1140 to $1260 \mathrm{~kg} / \mathrm{m}^{3}$ and speeds of sound from 1540 to $1600 \mathrm{~m} / \mathrm{s}$ were tried.

\subsection{Time domain signal reconstruction}

Previous studies of scattering from individual cells ${ }^{7,8}$ focused on the analysis of experimental results in the frequency domain through the BSTF. The analysis of data in time domain provides additional insight into scattering behavior of objects, such as ringing patterns and ring-down time. In this work, experimental and theoretical backscatter signals from sea urchin oocytes are presented and compared in both time and frequency domains. Given the experimental transmitted pulse and the theoretical backscatter frequency responses, the constructed time domain echo is given by

$$
r_{\text {theor }}(t)=\mathcal{F}^{-1}\left\{R_{\text {ref }}(\omega) \operatorname{BSTF}_{\text {theor }}(\omega)\right\}
$$

where, $\mathcal{F}^{-1}\{\}$ is the inverse Fourier transform and $\operatorname{BSTF}_{\text {theor }}(\omega)$ is the scattered echo of a homogeneous sphere insonified by a plane wave of unit amplitude. ${ }^{10,7}$ 

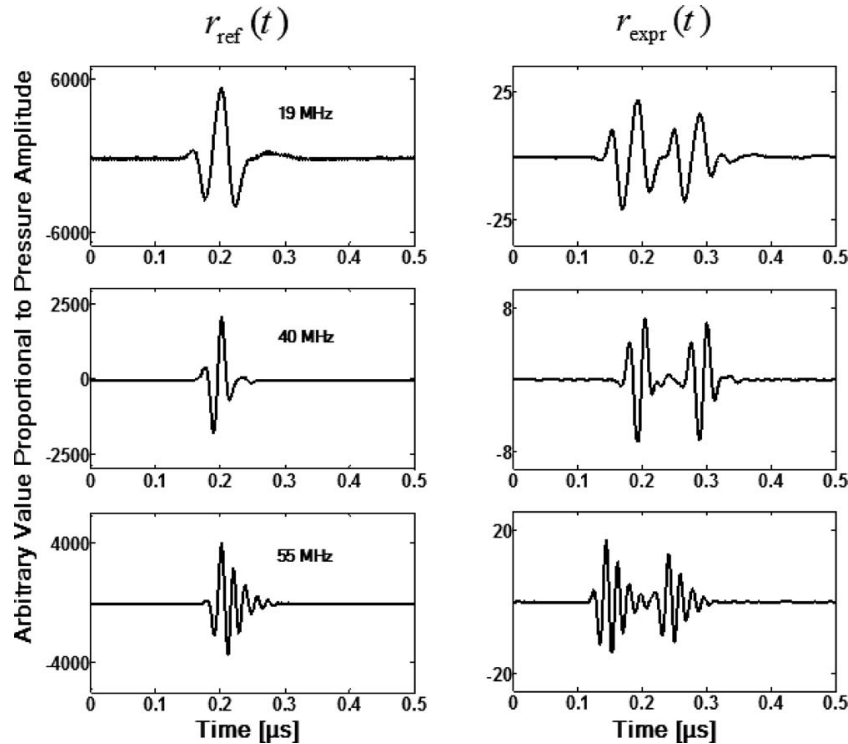

Fig. 2. Average backscatter echoes from individual sea urchin oocytes and their corresponding incident pulses from three transducers: 20, 40, and $80 \mathrm{MHz}$ excited at 19, 40, and $55 \mathrm{MHz}$, respectively.

\section{Results}

Figure 1 shows the B scan of sparse suspension of sea urchin oocytes in seawater at $40 \mathrm{MHz}$. Each pair of bright bands corresponds to the backscatter from a single oocyte. For each transducer, the average backscatter echoes from individual sea urchin oocytes (using the method described previously) and their corresponding average incident pulses are presented in Fig. 2. The measured backscatter frequency responses within a range that corresponds to the $-6 \mathrm{~dB}$ bandwidths of each transducer are plotted in Fig. 3. A theoretical density of $1198 \mathrm{~kg} / \mathrm{m}^{3}$ and a speed of sound of $1573 \mathrm{~m} / \mathrm{s}$ for the oocyte were found to have the best fit to experimental data. The theoretical frequency backscatter response of a fluid sphere $(d=75 \mu \mathrm{m}, c=1573 \mathrm{~m} / \mathrm{s}, \rho$ $\left.=1198 \mathrm{~kg} / \mathrm{m}^{3}\right)$ is also shown in Fig. 3. The average experimental and reconstructed signals from individual sea urchins oocytes from the $40 \mathrm{MHz}$ transducer (which produced the most distinct double bright bands per oocyte) are presented in Fig. 4.

\section{Discussion and Conclusions}

There is a very good agreement in the locations of maxima and minima, as shown in Fig. 3. The difference has been measured to be less than $1 \%$ "on average" between the theoretical fre-

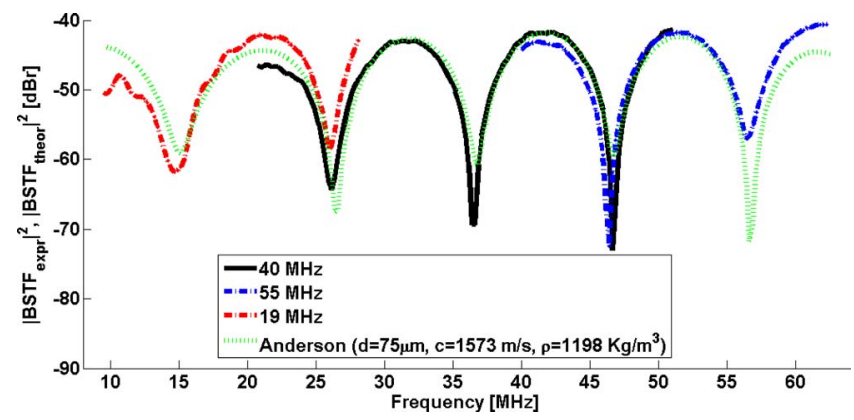

Fig. 3. (Color online) Theoretical (fluid sphere model parameters: $d=75 \mu \mathrm{m}, c=1573 \mathrm{~m} / \mathrm{s}, \rho=1198 \mathrm{~kg} / \mathrm{m}^{3}$ ) and measured backscatter frequency responses of single sea urchin oocytes in seawater subject to three incident pulses from three transducers: 20,40 , and $80 \mathrm{MHz}$ excited at 19,40 , and $55 \mathrm{MHz}$, respectively. 


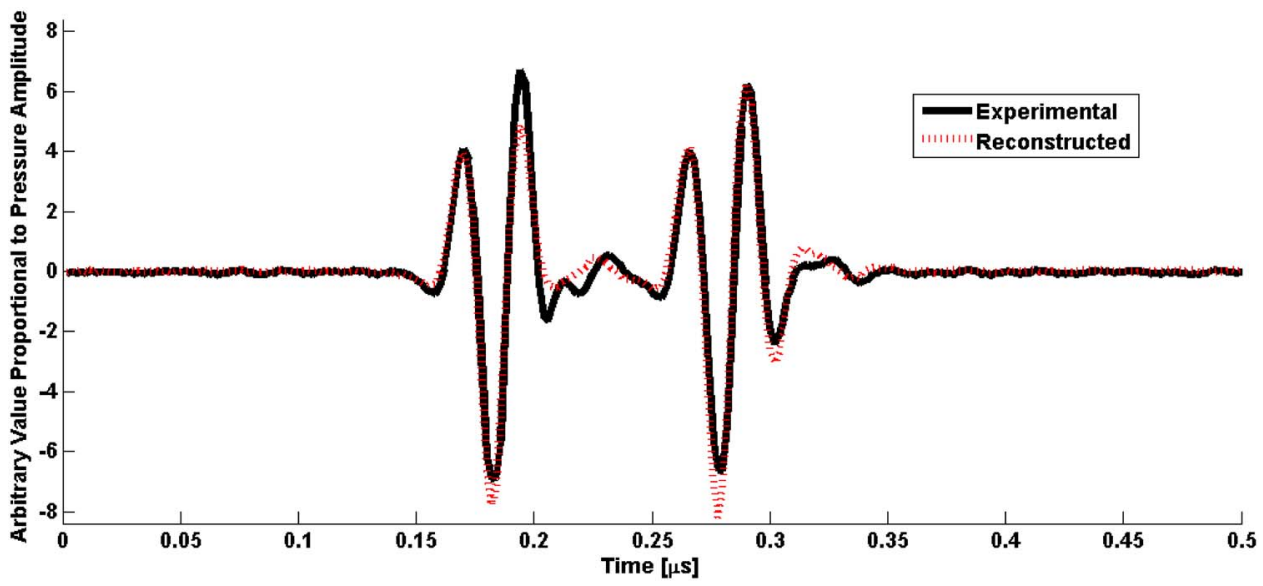

Fig. 4. (Color online) Average experimental and reconstructed (given the experimental transmitted pulse and the theoretical backscatter frequency responses) echoes from individual sea urchin oocytes at $40 \mathrm{MHz}$. Note that the reflection off the front and the back surface is similar to the incident pulse and there is no phase reversal at the back surface.

quency response of a fluid sphere and the measured backscatter frequency response of individual sea urchin oocytes. The presence of many ripples in the experimental data collected using the $20 \mathrm{MHz}$ transducer may be due to relatively low number of rf lines left (five of them) after discarding inappropriate scans (as described in Sec. 2). This suggests that most of the oocytes in the solution were not located within the $-6 \mathrm{~dB}$ focal volume of the transducer in the particular experiment. The overall agreement shown in Fig. 3 suggests that sea urchin oocytes can be best modeled as fluid sphere. These findings are not surprising since sea urchin oocytes have no membrane delimited nucleus and have a spherical shape (inset of Fig. 1).

The incident pulses and backscatter echoes for each transducer (Fig. 2) reveal that sea urchins are very weak scatterers [peak of echoes from quartz plate were $\sim 300$ times $(-49.5 \mathrm{~dB})$ larger than that of the backscatter echoes]. No ringing pattern was observed which indicates the absence of detectable shear waves inside the oocytes, a further indication of their fluidlike nature. Scattering seems to occur off the front and back surfaces of the sea urchin oocytes, as shown in the B-scan image (Fig. 1). The backscatter echo is composed of two pulses which can be easily distinguished for the $40 \mathrm{MHz}$ transducer. The peak-to-peak separation of the latter is $0.096 \mu$ s which corresponds to a distance of $151 \mu \mathrm{m}$ (the distance was found by multiplying the peak-to-peak separation by the speed of sound in the oocyte) or approximately twice the mean diameter of the oocytes. These findings further strengthen the argument that the oocytes can be modeled as fluid spheres.

A good agreement was found in the time domain between the experimental and reconstructed echoes from individual oocytes, as shown in Fig. 4. The maxima/minima that are larger than \pm 1 a.u. align temporally with no measureable error and their values agree to an average error of $24 \%$. In macroscopic models of wave propagation, a phase inversion occurs in the reflection at the boundary between two media when a wave travels from a region with higher acoustic impedance to lower impedance. The experimental rf data (Fig. 4) do not exhibit this phase reversal at the back surface of the oocyte (where the wave travels from higher to lower impedance). These results suggest that, although scattering mainly occurs at the surrounding medium-oocyte interfaces, a complete scattering model, such as that of Anderson, is needed to predict the scattered field from spherical objects.

In conclusion, the backscatter response from sea urchin oocytes were measured and compared to theoretical predictions for a fluid sphere (Anderson model). Very good agreement was found between the experimental and theoretical results in the frequency domain (difference less than $1 \%$ on average) suggesting that the backscatter response from a sea urchin oocyte is 
best modeled as a fluid sphere. This work confirms the fluid behavior of the non-nucleated biological specimens used in this study at high ultrasound frequencies. It also establishes an objective methodology to determine the appropriate theoretical model of the properties of individual cells as ultrasound scatterers. Future work will include the use of this methodology to test a hypothesis that acute myeloid leukemia cells, whose nucleus to cell volume ratio equals to 0.5 , are best modeled as an elastic nucleus surrounded by a fluid cytoplasm. Computational methods (such as the finite-element analysis) will be used to solve the theoretical model.

\section{Acknowledgments}

The authors would like to thank Mr. Zenon Harley and Dr. Homayoun Vaziri for their expertise and guidance and providing the sea urchin oocytes used in this study. This project was funded by the Canadian Institutes of Health Research (Grant No. 79447). This research was undertaken, in part, thanks to funding from the Canada Research Chairs Program awarded to M.C.K. O.F. is the recipient of an Ontario Graduate Scholarship. Funding to purchase the equipment was provided by the Canada Foundation for Innovation, the Ontario Ministry of Research and Innovation and Ryerson University.

\section{References and Links}

${ }^{1}$ J. W. Hunt, A. E. Worthington, A. Xuan, M. C. Kolios, G. J. Czarnota, and M. D. Sherar, "A model based upon pseudo regular spacing of cells combined with the randomisation of the nuclei can explain the significant changes in high-frequency ultrasound signals during apoptosis," Ultrasound Med. Biol. 28, 217-226 (2002).

${ }^{2}$ A. S. Tunis, G. J. Czarnota, A. Giles, M. D. Sherar, J. W. Hunt, and M. C. Kolios, "Monitoring structural changes in cells with high-frequency ultrasound signal statistics," Ultrasound Med. Biol. 31, 1041-1049 (2005).

${ }^{3}$ M. L. Oelze, W. D. O’Brien, Jr., J. P. Blue, and J. F. Zachary, "Differentiation and characterization of rat mammary fibroadenomas and 4T1 mouse carcinomas using quantitative ultrasound imaging," IEEE Trans. Med. Imaging 23, 764-771 (2004).

${ }^{4}$ G. J. Czarnota, M. C. Kolios, H. Vaziri, S. Benchimol, F. P. Ottensmeyer, M. D. Sherar, and J. W. Hunt, "Ultrasonic biomicroscopy of viable, dead and apoptotic cells," Ultrasound Med. Biol. 23, 961-965 (1997).

${ }^{5}$ J. F. R. Kerr, A. H. Wyllie, and A. R. Currie, "Apoptosis-Basic biological phenomenon with wide-ranging implications in tissue kinetics," Br. J. Cancer 26, 239-257 (1972).

${ }^{6}$ M. C. Kolios, G. J. Czarnota, M. Lee, J. W. Hunt, and M. D. Sherar, "Ultrasonic spectral parameter characterization of apoptosis," Ultrasound Med. Biol. 28, 589-597 (2002).

${ }^{7}$ R. E. Baddour, M. D. Sherar, J. W. Hunt, G. J. Czarnota, and M. C. Kolios, "High-frequency ultrasound scattering from microspheres and single cells," J. Acoust. Soc. Am. 117, 934-943 (2005).

${ }^{8}$ R. E. Baddour and M. C. Kolios, "The fluid and elastic nature of nucleated cells: Implications from the cellular backscatter response," J. Acoust. Soc. Am. 121, EL16-EL22 (2007).

${ }^{9}$ K. V. MacKenzie, "Nine-term equation for sound speed in the oceans," J. Acoust. Soc. Am. 70, 807-812 (1981).

${ }^{10}$ V. C. Anderson, "Sound scattering from a fluid sphere," J. Acoust. Soc. Am. 22, 426-431 (1950). 